\title{
Commentary: A novel infection- and inflammation-associated molecular signature in peripheral blood of myasthenia gravis patients
}

\author{
Claudia Barzago', Pia Bernasconi', Raffaele A. Calogero², Carlo Antozzi', Francesca Zolezzi ${ }^{3}$, Renato \\ Mantegazza ${ }^{1 *}$ and Lucia Mori ${ }^{4 *}$ \\ Neurology IV - Neuroimmunology and Neuromuscular Diseases Unit, Foundation Neurological Institute "Carlo Besta", 20133 Milan, Italy \\ ${ }^{2}$ Molecular Biology Center, Department of Molecular Biotechnology and Health Sciences, University of Turin, 10126 Turin, Italy \\ ${ }^{3}$ GALDERMA R\&D, 06902 Sophia Antipolis Cedex, France \\ ${ }^{4}$ Experimental Immunology, Department of Biomedicine, University Hospital Basel and University of Basel, 4031, Basel, Switzerland
}

Article Info

\section{Article Notes}

Received: September 22, 2016

Accepted: October 17, 2016

\section{${ }^{*}$ Correspondence:}

Dr. Lucia Mori,

Experimental Immunology, Department of Biomedicine, University Hospital Basel and University of Basel, 4031, Basel, Switzerland, Email: lucia.mori@unibas.ch

"Correspondence: Dr. Renato Mantegazza,

Neurology IV - Neuroimmunology and Neuromuscular Diseases Unit, Foundation Neurological Institute "Carlo Besta", 20133 Milan, Italy, Email: renato.mantegazza@istituto-besta.it

(c) 2016 Mori L \& Mantegazza R. This article is distributed under the terms of the Creative Commons Attribution 4.0 International License

\section{ABSTRACT}

Early-onset acetylcholine receptor-positive myasthenia gravis is the most studied and better-characterized clinical subgroup of myasthenia gravis. Here we discuss the results of the first comprehensive and unbiased transcriptome sequencing analysis performed on circulating cells of a clinically homogeneous cohort of patients affected by this disease form.

\section{Background}

The revolution of next-generation sequencing (NGS) technologies, occurred more than 10 years ago, instantly captured scientists' attention since the massive sequencing performances can accelerate new discoveries in genomic science allowing researchers to deeply explore genomics, transcriptomics, and epigenomics mechanisms involved in human diseases. In particular, the relevance of NGS applications in the field of autoimmune disorders is constantly emerging ${ }^{1,2}$. For instance, by using RNA-sequencing (RNA-seq), Tsoi et al. ${ }^{1}$ discovered tissue-specific long non-coding RNAs (lncRNAs) in the skin of patients affected by psoriasis and several lncRNAs with putative immunological functions, suggesting their contribution to psoriasis pathogenesis. In addition, RNA-seq applied on peripheral blood monocytes isolated from systemic lupus erythematosus (SLE) patients revealed that full-length transcripts of interferon regulatory factor 5, a gene involved in immune reactions and inflammation, had a different expression profile as compared to healthy donor specimens ${ }^{2}$. These and other studies indicate that NGS is an in-depth tool for the analysis of transcriptome, which in turn could reveal molecular alterations potentially involved in disease pathogenesis ${ }^{3}$.

In our study ${ }^{4}$ RNA-seq was used to investigate the peripheral transcriptome in patients with myasthenia gravis (MG), an autoimmune disease of the neuromuscular junction (NMJ). $)^{5}$ is caused by the abnormal production of autoantibodies targeting the acetylcholine receptor $(\mathrm{AChR})^{6}$ in roughly $80 \%$ of patients. Other autoantibody protein targets, such as muscle kinase receptor and lipoprotein receptor-related protein 4 , have also been detected in MG patients ${ }^{7-9}$. The thymus is generally accepted as being the key organ in which the autosensitisation process takes place in AChR-positive MG patients (AChR-MG), who frequently show 
anatomical and physiological thymic changes including hyperplasia and thymoma ${ }^{10-12}$. Several studies showed that MG thymus is characterized by a chronic inflammatory status, probably related to the activation via Toll-like receptor (TLR) engagement of innate immunity against persistent viral infections, which may interfere with immunological tolerance and lead to autoimmunity onset or perpetuation ${ }^{13-16}$. A deep characterization of the molecular alterations connecting intra-thymic MG pathogenesis with the peripheral autoimmune responses explicated at the NMJ is however poorly known.

MG is a complex and heterogeneous disorder presenting clinical variations in age at onset, autoantibody specificity, disease severity, and thymic involvement. Its heterogeneity also manifests as variable patients' responses to the therapeutic treatments (e.g. acetylcholinesterase inhibitors, azathioprine, steroids, and thymectomy), which lead to complete stable remission in only a proportion of patients, thus underlying the need to identify the exact molecular events contributing to the disease $\mathrm{e}^{17,18}$.

Identification of peripheral dysregulated transcripts and microRNAs in AChR-EOMG

Through RNA-seq we analysed the transcriptional pattern of peripheral blood mononuclear cells (PBMCs) of the better-characterized MG clinical subgroup, the AChR$M G$ patients with a disease onset under the age of 50 years (AChR-EOMG). We studied a clinically homogenous group of MG patients consisting in 19 patients untreated or treated with non-steroid therapy, with the aim of reducing variability between among patients and gaining knowledge about the molecular changes arising in the periphery of AChR-EOMG patients.

The use of RNA-seq technology, never before applied in MG, allowed us to identify 128 annotated coding transcripts and 229 lncRNAs, including 9 microRNA precursors (premiRNAs), as dysregulated in AChR-EOMG PBMCs compared to age- and gender-matched healthy donors. We focused on the dysregulated coding transcripts and miRNAs, therefore future studies are needed to clarify the relevance of the identified dysregulated lncRNAs in MG.

Bioinformatics analysis showed that infectious disease', 'inflammatory disease' and 'inflammatory response' functional categories were significantly enriched in dysregulated transcripts. Validation of selected coding transcripts supported the evidence that PBMCs of our cohort of AChR-EOMG patients presented altered 'infectious-related' and 'inflammatory-related' transcripts, including Charcot-Leyden crystal galectin $(C L C)$, eukaryotic translation termination factor 1 (ETF1), interleukin 4 (IL4), nuclear factor of kappa light polypeptide gene enhancer in B-cells 2 (NFKB2), polo-like kinase 3 (PLK3), and protein phosphatase 1 regulatory subunit 15A (PPP1R15A) for the former group, and ATP-binding cassette sub-family A member 1 (ABCA1), fused in sarcoma (FUS), and v-rel reticuloendotheliosis viral oncogene homolog $\mathrm{B}(R E L B)$ for the latter. Of note, none of the patients, except one, had a chronic viral infection at time of blood collection, suggesting that the dysregulated molecules we identified were not merely influenced by a random event but might have a pathological meaning in AChR-EOMG. Based on literature findings, the involvement of $I L 4$ and PPP1R15A in MG is of particular interest ${ }^{19,20}$. IL4, a multiple functional cytokine, might have a protective action in the experimental model of MG participating in the prevention of AChR-specific autoimmune response ${ }^{19}$. Moreover, Uzawa et al. ${ }^{20}$ showed that IL4 serum levels were decreased in MG patients compared to healthy controls. In agreement with these observations, we found a down-regulation of IL4 transcript in AChR-EOMG PBMCs, thus further supporting the critical role of $I L 4$ in MG that could be hypothetically linked to a mechanism by which the reduction of the protective IL4 may contribute to disease progression in periphery. With regards to $P P P 1 R 15 A$, a DNA damage-inducible protein, it is found to be essential for the TLR3-mediated cytokine release (e.g. IFN- $\beta$ ) during viral infection in mice $^{21}$. Interestingly, it has been recently showed that the activation of IFN- $\beta$-mediated TLR3-dependent signalling pathway increased the expression of AChR $\alpha$ subunit in thymic epithelial cell cultures, as well as the production of anti-AChR autoantibodies in experimental autoimmune MG mice ${ }^{13}$. In our study, we observed an up-regulation of PPP1R15A transcript in AChR-EOMG PBMCs that might contribute to skew the inflammatory fine balance, probably via TLR alterations, thus participating in the maintenance of the autoimmune reaction in periphery.

Our overall transcriptome results are in line with literature data showing a key role of infection-related and inflammatory pathways in the intra-thymic MG pathogenesis ${ }^{13,15,16,22}$. Though further studies are indispensable to explore the functional role of the dysregulated molecules we identified, our data disclosed the possible contribution of a new molecular signature in MG.

MiRNAs are small non-coding RNAs having a posttranscriptional regulatory function of the gene expression that may potentially influence thousands of target proteincoding genes; therefore they may have a relevant impact in almost all biological processes ${ }^{23,24}$. A critical involvement of miRNA dysregulation has already been reported in several autoimmune disorders, including rheumatoid arthritis and $\mathrm{SLE}^{25,26}$. In MG, the contribution of miRNAs is rapidly emerging, although little is known about how their altered mechanisms of action underlie disease pathogenesis. Of note, recent studies identified miR21-5p and miR-150-5p as potential MG-specific serum 
biomarkers, since their circulating levels were increased in MG patients as compared with patients affected by other autoimmune disorders (e.g. psoriasis) and to healthy donors $^{27,28}$. Our RNA-seq study in PBMCs showed that miR612, miR-3654, miR-3651, and pre-miR-3651 levels were increased in AChR-EOMG patients as compared to controls. These miRNAs have been recently annotated, and the lack of findings about their exact role in human diseases makes it difficult to contextualise them in a biological prospective, especially for the high complexity of miRNAmRNA target interactions. Nonetheless, we predicted interactions between miR-612 and two putative target transcripts, $H R H 4$ and $A K A p 12$, that our data revealed to be differentially expressed in AChR-EOMG PBMCs. Specifically, we found an anti-correlation between the expression levels of miR-612 (up-regulated) and those of HRH4 and AKAp12 (down-regulated), that suggested the involvement of these dysregulated miRNA-mRNA pairs in MG.

\section{Conclusions}

Our findings revealed a novel dysregulated signature in peripheral blood cells of AChR-EOMG patients. In particular, we showed that 'infection-' and 'inflammationrelated' molecules may contribute to the perpetuation of the peripheral autoimmune responses in AChR-EOMG. This transcriptome analysis provided new knowledge about the molecular features underlying MG pathogenesis and paved the way to the development of target-specific therapeutic interventions in the disease. Future studies will describe the functional role of the molecules we found differentially expressed. Along with the described molecules, our highthroughput approach produced a massive amount of data, which may be hardly addressed by classical biological techniques. Hence, system biology approach will help understanding and, possibly, elucidating the intrinsic high complexity of MG.

\section{Acknowledgements}

C.B. is supported by the Italian Ministry of Foreign Affairs and International Cooperation (Agreement on Scientific, Technological and Industrial Cooperation between Italy and Israel, 2015).

\section{References}

1. Tsoi LC, Iyer MK, Stuart PE, Swindell WR, Gudjonsson JE, Tejasvi T, et al. Analysis of long non-coding RNAs highlights tissue-specific expression patterns and epigenetic profiles in normal and psoriatic skin. Genome Biol. 2015; 16:24.

2. Stone RC, Du P, Feng D, Dhawan K, Rönnblom L, Eloranta ML, et al. RNA-Seq for enrichment and analysis of IRF5 transcript expression in SLE. PLoS One. 2013; 8(1): e54487.

3. Ma Y, Shi N, Li M, Chen F, Niu H. Applications of Next-generation Sequencing in systemic autoimmune diseases. Genomics Proteomics Bioinformatics. 2015; 4: 242-249.
4. Barzago C, Lum J, Cavalcante P, Srinivasan KG, Faggiani E, Camera G, et al. A novel infection- and inflammation-associated molecular signature in peripheral blood of myasthenia gravis patients. Immunobiology. 2016; 221(11): 1227-1236.

5. Berrih-Aknin S, Le Panse R. Myasthenia gravis: a comprehensive review of immune dysregulation and etiological mechanisms. J Autoimmun. 2014; 52: 90-100.

6. Cavalcante P, Bernasconi P, Mantegazza R. Autoimmune mechanisms in myasthenia gravis. Curr Opin Neurol. 2012; 25(5): 621-629.

7. Hoch W, McConville J, Helms S, Newsom-Davis J, Melms A, Vincent A. Auto-antibodies to the receptor tyrosine kinase MuSK in patients with myasthenia gravis without acetylcholine receptor antibodies. Nat Med. 2001; 7(3): 365-368.

8. Higuchi O, Hamuro J, Motomura M, Yamanashi Y. Autoantibodies to low-density lipoprotein receptor-related protein 4 in myasthenia gravis. Ann Neurol. 2011; 69(2): 418-422.

9. Zhang B, Tzartos JS, Belimezi M, Ragheb S, Bealmear B, Lewis RA, et al. Autoantibodies to lipoprotein-related protein 4 in patients with double-seronegative myasthenia gravis. Arch Neurol. 2012; 69(4): 445-451.

10. Marx A, Schultz A, Wilisch A, Nenninger R, Müller-Hermelink HK. Myasthenia gravis. Verh Dtsch Ges Pathol. 1996; 80: 116-126.

11. Mantegazza R, Baggi F, Bernasconi P, Antozzi C, Confalonieri P, Novellino L, et al. Video-assisted thoracoscopic extended thymectomy and extended transsternal thymectomy (T-3b) in non-thymomatous myasthenia gravis patients: remission after 6 years of follow-up. J Neurol Sci. 2003; 212(1-2): 31-36.

12. Berrih-Aknin S, Ragheb S, Le Panse R, Lisak RP. Ectopic germinal centers, BAFF and anti-B-cell therapy in myasthenia gravis. Autoimmun Rev. 2013; 12(9): 885-893.

13. Cufi P, Dragin N, Weiss JM, Martinez-Martinez P, De Baets MH, Roussin $\mathrm{R}$, et al. Implication of double-stranded RNA signaling in the etiology of autoimmune myasthenia gravis. Ann Neurol. 2013; 73(2): 281-293.

14. Cavalcante P, Galbardi B, Franzi S, Marcuzzo S, Barzago C, Bonanno S, et al. Increased expression of Toll-like receptors 7 and 9 in myasthenia gravis thymus characterized by active Epstein-Barr virus infection. Immunobiology. 2016; 221(4): 516-527.

15. Cavalcante P, Cufi P, Mantegazza R, Berrih-Aknin S, Bernasconi P, Le Panse R. Etiology of myasthenia gravis: innate immunity signature in pathological thymus. Autoimmun Rev. 2013; 12(9): 863-874.

16. Cordiglieri C, Marolda R, Franzi S, Cappelletti C, Giardina C, Motta T, et al. Innate immunity in myasthenia gravis thymus: pathogenic effects of Toll-like receptor 4 signaling on autoimmunity. J Autoimmun. 2014; 52:74-89.

17. Baggi F, Andreetta F, Maggi L, Confalonieri P, Morandi L, Salerno F, et al. Complete stable remission and autoantibody specificity in myasthenia gravis. Neurology. 2013; 80(2): 188-195.

18. Mantegazza R, Bonanno S, Camera G, Antozzi C. Current and emerging therapies for the treatment of myasthenia gravis. Neuropsychiatr Dis Treat. 2011; 7:151-160.

19. Ostlie N, Milani M, Wang W, Okita D, Conti-Fine BM. Absence of IL-4 facilitates the development of chronic autoimmune myasthenia gravis in C57BL/6 mice. J Immunol. 2003; 170(1): 604-612.

20. Uzawa A, Kawaguchi N, Himuro K, Kanai T, Kuwabara S. Serum cytokine and chemokine profiles in patients with myasthenia gravis. Clin Exp Immunol. 2014; 176(2): 232-237.

21. Clavarino G, Cláudio N, Couderc T, Dalet A, Judith D, Camosseto V, et al. Induction of GADD3 4 is necessary for dsRNA-dependent interferon- $\beta$ 
production and participates in the control of Chikungunya virus infection. PLoS Pathog. 2012; 8(5): e1002708.

22. Cavalcante P, Maggi L, Colleoni L, Caldara R, Motta T, Giardina C, et al. Inflammation and epstein-barr virus infection are common features of myasthenia gravis thymus: possible roles in pathogenesis. Autoimmune Dis. 2011; 213092.

23. Towler BP, Jones CI, Newbury SF. Mechanisms of regulation of mature miRNAs. Biochem Soc Trans. 2015; 43(6): 1208-1214.

24. Saetrom P, Heale BS, Snøve O Jr, Aagaard L, Alluin J, Rossi JJ. Distance constraints between microRNA target sites dictate efficacy and cooperativity. Nucleic Acids Res. 2007; 35(7): 2333-2342.

25. Alsaleh G, Suffert G, Semaan N, Juncker T, Frenzel L, Gottenberg JE, et al.
Bruton's tyrosine kinase is involved in miR-346-related regulation of IL-18 release by lipopolysaccharide-activated rheumatoid fibroblastlike synoviocytes. J Immunol. 2009; 182(8): 5088-5097.

26. Tang Y, Luo X, Cui H, Ni X, Yuan M, Guo Y, et al. MicroRNA-146A contributes to abnormal activation of the type I interferon pathway in human lupus by targeting the key signaling proteins. Arthritis Rheum. 2009; 60(4): 1065-1075.

27. Punga T, Le Panse R, Andersson M, Truffault F, Berrih-Aknin S, Punga AR. Circulating miRNAs in myasthenia gravis: miR-150-5p as a new potential biomarker. Ann Clin Transl Neurol. 2014; 1(1): 49-58.

28. Punga AR, Andersson M, Alimohammadi M, Punga T. Disease specific signature of circulating miR-150-5p and miR-21-5p in myasthenia gravis patients. J Neurol Sci. 2015; 356(1-2): 90-96. 